\title{
Comparison of High-Dose versus Low-Dose Aspirin in the Management of Kawasaki Disease
}

\author{
Aliakbar Rahbarimanesh • Mozhgan Taghavi-Goodarzi • \\ Payam Mohammadinejad • Javad Zoughi • \\ Jalalaldin Amiri • Kasra Moridpour
}

Received: 2 April 2013 / Accepted: 21 March 2014 / Published online: 8 April 2014

(C) Dr. K C Chaudhuri Foundation 2014

To the Editor: Kawasaki disease (KD) is a vasculitis of an unknown origin, involving the small and medium size vessels $[1,2]$. Standard treatment for acute phase of KD introduced by American Heart Association (AHA) and American Academy of Pediatrics (AAP) includes intravenous immunoglobulin (IVIG) therapy with a single dose of $2 \mathrm{~g} / \mathrm{kg}$ plus oral administration of aspirin at high-dose of $80-100 \mathrm{mg} / \mathrm{kg} / \mathrm{d}[3,4]$.

During the acute phase of Kawasaki disease, Group 1 (control group), consisting of 27 children, received aspirin at a daily dose of $80-100 \mathrm{mg} / \mathrm{kg} / \mathrm{d}$ for $14 \mathrm{~d}$ after the onset. Highdose aspirin was then changed to low-dose (3-5 mg/ $\mathrm{kg} / \mathrm{d}$ ) for another 6-8 wk. Group 2 (study group) consisting of 42 children received aspirin at a daily dose of $3-5 \mathrm{mg} / \mathrm{kg} / \mathrm{d}$ for $8-10 \mathrm{wk}$ if no coronary artery aneurism was observed in echocardiography. All patients also received the standard IVIG therapy with a single dose of $2 \mathrm{~g} / \mathrm{kg}$.

The mean duration of fever after the therapy was $41.96 \pm$ $19.63 \mathrm{~h}$ in Group 1 and $46.00 \pm 50.49 \mathrm{~h}$ in Group $2(P=0.694)$. Persistent fever was observed in $14.8 \%$ of cases in Group 1 and $11.9 \%$ of cases in Group $2(P=0.729)$. Duration of the hospital stay was $6 \pm 1.3 \mathrm{~d}$ in Group 1 and $6.36 \pm 2.80 \mathrm{~d}$ in Group $2(P=0.540)$. There was $1(4 \%)$ and 2 cases $(5.3 \%)$ of

\footnotetext{
A. Rahbarimanesh $\cdot$ M. Taghavi-Goodarzi $(\bowtie) \cdot J$. Zoughi •

J. Amiri $\cdot$ K. Moridpour

Department of Pediatric Infectious Diseases, Bahrami Children

Hospital, Tehran University of Medical Sciences, Shaheed Kiani St., Damavand Ave., Tehran 14194, Iran

e-mail: taghavimozhgan@gmail.com

P. Mohammadinejad

Research Center for Immunodeficiencies, Pediatrics Center of Excellence, Children's Medical Center, Tehran University of Medical Sciences, Tehran, Iran
}

new coronary artery aneurism (CAA) formation in Group 1 and Group 2 respectively $(P=1.000)$.

The logic behind the use of aspirin regimen in KD is the probable benefits of its anti-inflammatory and anti-platelet effects at high and low dosages respectively [5]. According to the result of this study, it is most likely that the antiinflammatory dosage of aspirin has no advantage over the anti-platelet dosage regarding the duration of fever and days of hospital stay. This study was not powered enough to assess the differences between the two regimens regarding the CAA formation. Further studies with higher number of cases are needed to compare the effectiveness of high and low doses of aspirin in preventing CAA formation.

\section{Conflict of Interest None.}

Role of Funding Source Tehran University of Medical Sciences supported the study.

\section{References}

1. Freeman AF, Shulman ST. Kawasaki disease: summary of the American Heart Association guidelines. Am Fam Physician. 2006;74:1141-8.

2. Hsieh KS, Weng KP, Lin CC, Huang TC, Lee CL, Huang SM. Treatment of acute Kawasaki disease: aspirin's role in the febrile stage revisited. Pediatrics. 2004;114:689-93.

3. Brogan PA, Bose A, Burgner D, Shingadia D, Tulloh R, Michie C, et al. Kawasaki disease: an evidence based approach to diagnosis, treatment, and proposals for future research. Arch Dis Child. 2002;86:286-90.

4. Shin JI, Lee JS, Choi JY, Kim DS, Girish M, Subramaniam. Refractory Kawasaki disease: infliximab or methotrexate therapy? Indian J Pediatr. 2009;76:1184. author reply 1184

5. Burns JC, Glode MP. Kawasaki syndrome. Lancet. 2004;364:533-44. 